\title{
ESPECIFICIDADE DE HOSPEDEIRO: PADRÕES ECOLÓGICOS DE INSETOS FITÓFAGOS EM ECOSSISTEMAS TROPICAIS
}

\author{
Milena de Sousa Nascimento ${ }^{1,2 *} \&$ Ricardo Ferreira Monteiro ${ }^{1,2}$ \\ ${ }^{1}$ Laboratório de Ecologia de Insetos, Depto. de Ecologia, Inst. de Biologia, Universidade Federal do Rio de Janeiro (UFRJ). Ilha do Fundão. Rio de \\ Janeiro, Brasil. \\ ${ }^{2}$ Programa de Pós-Graduação em Ecologia, Inst. de Biologia, UFRJ. Ilha do Fundão. CEP: 21941-590, Caixa Postal: 68020. Rio de Janeiro, Brasil. \\ *E-mail: nascimentoms@biologia.ufrj.br
}

\section{RESUMO}

Estimativas de diversidade global de insetos apresentam uma grande amplitude de variação, o que reflete a enorme falta de conhecimento a respeito da especificidade de hospedeiro de insetos fitófagos, principalmente na região tropical. O objetivo dessa revisão é avaliar o estado de conhecimento dos padrões de especificidade de hospedeiro de insetos fitófagos na região tropical e, assim, direcionar o rumo das atuais tendências das estimativas sobre diversidade global de insetos. Trabalhos recentes apontam para resultados opostos em relação à especificidade de insetos fitófagos tropicais, porém ainda são escassos os trabalhos sobre especificidade na região tropical e nos seus diferentes biomas. Uma vez que a riqueza de espécies é bem maior nos trópicos, mas o conhecimento sobre a ecologia dos insetos é ainda pequeno nessa região, faltam levantamentos e estudos que permitam avaliar melhor as tendências das estimativas de diversidade global.

Palavras-chave: Especificidade de hospedeiro, insetos fitófagos, região tropical.

\begin{abstract}
HOST SPECIFICITY: ECOLOGICAL PATTERNS OF PHYTOPHAGOUS INSECTS IN TROPICAL ECOSYSTEMS. There is considerable variation between different estimates of global insect species diversity, and one of the causes for this is a paucity of knowledge about the host specificity of phytophagous insects, chiefly from tropical regions. Thus, the objective of the present review is to assess the state of knowledge about host specificity of tropical phytophagous insects while discussing the current trends of estimates of global insect diversity. Some recent publications present conflicting records of the host specificity of phytophagous insects, and studies dealing with the matter in the many tropical biomes are still scarce. Given the great species richness in the tropics and the current limited knowledge about the ecology of the vast majority of tropical insects, a number of investigations and studies are needed for a better evaluation of the tendencies of the present global insect species diversity estimates.
\end{abstract}

Keywords: Host specificity, phytophagous insects, tropical region.

\section{INTRODUÇÃO}

Atualmente, existem diversas estimativas sobre o número de espécies de insetos existentes no planeta, variando de cinco a 80 milhões de espécies (Stork 1988, Hodkinson 1992). Os cálculos que levam a essas estimativas são baseados em alguns parâmetros, como a diversidade de espécies nos trópicos, a quantidade de insetos herbívoros e, ainda, a especificidade de hospedeiro dos insetos herbívoros. Essa grande amplitude de estimativas reflete a enorme falta de conhecimento sobre grupos de insetos que não são herbívoros e até sobre a especificidade dos insetos herbívoros (May 1988).
Altas estimativas de riqueza global de espécies de insetos partem das seguintes premissas: 1) que o número de espécies de plantas nos trópicos é bem maior do que na região temperada, 2) que a maior parte dos insetos é herbívora, 3) que o número de espécies de insetos herbívoros por planta hospedeira é muito alto nos trópicos e 4) que na região tropical a especificidade de hospedeiro é mais alta (Erwin 1982, Dyer et al. 2007). Esses estudos aceitam a hipótese de que o alto número de espécies na região tropical é explicado pela maior especificidade de hospedeiro dos insetos herbívoros nos trópicos, uma vez que um dos mecanismos que permite a co-existência de espécies que ocupam o mesmo nicho ou nichos semelhantes é 
a especialização (Hutchinson 1959), pois esta reduz a competição interespecífica e permite que as espécies competidoras co-existam no espaço e tempo. Desse modo, esses trabalhos prevêem uma relação direta entre a especialização e a riqueza de espécies num determinado ambiente.

No entanto, vários estudos sobre a especificidade dos insetos fitófagos mostram que esta não chega a valores tão altos nos trópicos. Assim, outros estudos apresentam estimativas mais baixas do número de espécies de insetos no planeta, partindo de premissas que existem mais espécies de plantas na região tropical, porém a especificidade dos insetos herbívoros não é maior nos trópicos do que na região temperada (Stork 1993, Odegaard 2000, Novotny et al. 2002). Desse modo, o principal fator responsável pela maior riqueza de insetos fitófagos na região tropical seria a maior diversidade de plantas hospedeiras nessa região (Novotny et al. 2006).

A grande diversidade de espécies de plantas nos trópicos é bem reconhecida. Barthlott et al. (1996), por exemplo, encontraram de cinco a 10 vezes mais espécies de plantas emuma área de $10 \mathrm{Km}^{2}$ nos trópicos do que em área equivalente na região temperada. Entretanto, a diferença latitudinal na especificidade de hospedeiro e no número de espécies de insetos por espécie de planta é mais difícil de ser determinada (Lewinsohn et al. 2005, Novotny \& Basset 2005). Na região temperada, os padrões de uso do hospedeiro por insetos fitófagos têm sido bem documentados nos últimos dois séculos (Quinn et al. 1997, Stireman et al. 2005). Somente nas duas últimas décadas, alguns grupos de pesquisadores têm desenvolvido estudos na região tropical, a partir de coleta e criação de insetos fitófagos, produzindo dados relevantes sobre associações de insetos em suas espécies de plantas hospedeiras (ver, por exemplo, Diniz \& Morais 1995, Novotny et al. 2002, Flinte et al. 2006). Entretanto, os dados obtidos até o momento não permitem afirmar se a maior parte dos herbívoros tropicais é especialista ou generalista (Novotny et al. 2006, Dyer et al. 2007, Novotny et al. 2007), o que dificulta o entendimento da co-existência de tamanha diversidade de insetos nos trópicos.

Por esses motivos, pesquisas que contribuam para o conhecimento das plantas hospedeiras utilizadas por insetos fitófagos são de extrema importância, uma vez que a amplitude de plantas hospedeiras de insetos fitófagos é um parâmetro essencial para estudos de biodiversidade global. Embora mais demorados, os estudos sobre insetos fitófagos centrados em espécies de plantas permitem a identificação das plantas hospedeiras, o estudo das distribuições temporal e espacial dos insetos, o conhecimento de aspectos biológicos e de interações das espécies (Lewinsohn et al. 2001). Além disso, estudos voltados para especificidade de insetos podem servir de suporte para teorias ecológicas e evolutivas, esclarecendo o papel da especialização ecológica na diversificação das espécies (Dyer et al. 2007).

Nesse sentido, o objetivo dessa revisão é avaliar o estado de conhecimento dos padrões de especificidade de hospedeiro de insetos fitófagos na região tropical, compará-los com padrões obtidos na região temperada e, assim, permitir conclusões sobre o rumo das atuais tendências das estimativas sobre diversidade global de insetos. Pretende-se também, através dos padrões de especificidade encontrados, explicar possíveis padrões de abundância de comunidades de insetos fitófagos.

\section{ESPECIFICIDADE DE HOSPEDEIRO DE IN- SETOS FITÓFAGOS NA REGIÃO TROPICAL}

Já na década de 60, alguns pesquisadores se empenhavam em desvendar os mistérios das relações entre insetos fitófagos e suas plantas hospedeiras. Thorsteinson (1960) acreditava que a escolha de uma determinada planta hospedeira ou de um grupo de plantas por um inseto fitófago era conduzida não só pela presença de substâncias atraentes nessas plantas, mas também pela presença de substâncias desagradáveis nas outras plantas. Em 1964, Ehrlich \& Raven, em trabalho sobre co-evolução de borboletas e suas plantas hospedeiras, concluíram que, em geral, as espécies de plantas hospedeiras servem de alimento, somente para um grupo ou grupos próximos filogeneticamente de espécies de borboletas. Os autores não acreditavam na evolução gradativa de insetos polífagos a partir de insetos mais especializados, assim como também não acreditavam na existência de uma planta hospedeira que fosse aceita universalmente por qualquer inseto. Para esses autores, as plantas e os insetos fitófagos evoluíram, em parte, um em resposta ao outro, criando entre esses organismos interações ecológicas específicas e essas interações seriam o principal fator responsável 
pela grande diversidade de espécies de insetos e de plantas observadas hoje na região tropical. Seguindo essa linha, Erwin (1982), em uma das mais famosas estimativas de riqueza global de espécies, calcula um total de 30 milhões de espécies de insetos no planeta, considerando que cada uma das 50.000 espécies de árvores do planeta hospedaria 165 espécies de besouros, sendo estes $40 \%$ das espécies de insetos. Entretanto, Hammond (1992), em revisão sobre estimativas de diversidade global, coloca o grupo dos insetos entre os domínios menos conhecidos em relação à sua riqueza de espécies, o que dificulta a obtenção de estimativas precisas dessa biodiversidade.

Nas décadas de 80 e 90, diversos trabalhos foram publicados na tentativa de esclarecer as relações entre insetos e plantas, trazendo cada vez mais conhecimento acerca da especificidade dos insetos fitófagos (Price 1984, Strong 1984, Diniz \& Morais 1995). A maior parte desses trabalhos apresenta resultados que confirmam a hipótese de que insetos fitófagos tropicais são mais especialistas do que os insetos fitófagos da região temperada. Janzen (1988), em estudo realizado na Costa Rica, concluiu que $90 \%$ das espécies de insetos eram oligófagas ou monófagas, ou seja, se alimentavam exclusivamente de uma família de plantas. Marquis (1991), estudando coleópteros e lepidópteros, também na Costa Rica, encontrou que cada espécie de inseto utilizava, em média, entre uma e quatro espécies de plantas hospedeiras. Por outro lado, na mesma época, alguns trabalhos mostram padrões de menor especificidade dos insetos fitófagos tropicais (Lopes 1995, Basset 1996).

Mais recentemente, estudos em diferentes localidades continuam encontrando resultados paradoxais. Nos últimos anos, diversos estudos sobre especificidade de hospedeiro de insetos fitófagos, desenvolvidos na Guiana e Papua Nova Guiné (Novotny et al. 2002, 2004, 2006, 2007), têm reforçado os padrões de especificidade mais baixos para insetos fitófagos tropicais. Seus resultados têm reduzido as estimativas da diversidade global de insetos para cerca de 4-5 milhões de espécies (Novotny et al. 2002). Esses estudos avaliam a especificidade de insetos fitófagos a partir de um grande conjunto de dados de registros de plantas hospedeiras utilizadas por insetos. Para isso, contam com a ajuda de parataxonomistas locais, treinados para coleta em campo, criação e montagem dos insetos em laboratório (Basset et al. 2000).
Em revisão sobre especificidade de insetos fitófagos tropicais, Novotny \& Basset (2005) observaram que apenas $27 \%$ das espécies de herbívoros se alimentavam de uma única espécie de planta, quando outras espécies de plantas do mesmo gênero ou família também estavam disponíveis no ambiente. As porcentagens aumentavam para 48 e $58 \%$ quando eram analisados insetos herbívoros se alimentando do mesmo gênero ou família de planta, respectivamente. No entanto, ao final da revisão, os autores concluíram ainda não ser possível uma definição sobre a especificidade de hospedeiro de insetos fitófagos tropicais. Nos seus dois últimos trabalhos, a equipe comparou a especificidade de insetos fitófagos coletados na região tropical e na região temperada (Novotny et al. 2006) e também analisou a diversidade beta de insetos fitófagos de florestas tropicais (Novotny et al. 2007). Nos resultados, os autores, além de não observarem diferenças significativas entre a especificidade de hospedeiro dos insetos fitófagos e nem entre o número de espécies de insetos fitófagos por espécie de planta nas duas regiões, encontraram uma baixa taxa de mudança espacial na composição de espécies de insetos (beta diversidade). Portanto, os autores concluíram que a especificidade de hospedeiro não difere latitudinalmente, refutando a hipótese de que na região tropical os recursos são mais partilhados entre os insetos fitófagos do que na região temperada, e sugeriram que o maior número de espécies de insetos nos trópicos é uma função direta do maior número de espécies de plantas nessa região. Essas observações concordam com outros trabalhos que encontraram beta diversidade baixa quando calculada através do contingente regional de espécies (Gaston \& Gauld 1993, Orr \& Haeuser 1996, Novotny et al. 2005).

Em trabalho publicado recentemente, Dyer et al. (2007) compilaram dados sobre espécies de lagartas coletadas em campo e criadas em laboratório, em oito localidades do continente americano, contemplando regiões temperadas, subtropicais e tropicais. Os resultados mostraram que a especificidade de hospedeiro diminui significativamente com o aumento da latitude, ou seja, insetos fitófagos na região tropical são mais específicos do que na região temperada. A análise da diversidade beta por esses autores sugere que esta é maior em florestas tropicais, intermediária em florestas subtropicais e menor em florestas temperadas, contradizendo os resultados encontrados por Novotny et al. (2007). 
A discrepância nos resultados desses trabalhos pode ser explicada por diferentes fatores, sendo um dos principais, as diferenças na metodologia utilizada por eles. O artigo de Novotny et al. (2007), mostra baixa diversidade beta nos trópicos e foi realizado em floresta pluvial homogênea, sem variação altitudinal, de tipo de solo e de composição da vegetação. Já Dyer et al. 2007 apresentam artigo que mostra alta diversidade beta nos trópicos e envolveu comparações de coletas realizadas em diferentes tipos de ambientes, com diferentes altitudes e diferentes composições vegetacionais, o que pode ter favorecido a alta taxa de variação na composição de espécies de insetos entre diferentes locais. Por outro lado, o trabalho de Novotny et al. (2006), que não mostra diferença entre as especificidades de insetos fitófagos nas duas regiões, possui tamanho amostral relativamente pequeno, limitando a comparação entre as duas regiões a apenas 14 espécies de plantas. Além disso, as diferenças nos resultados dos trabalhos podem ocorrer simplesmente pelas diferenças biológicas entre os diferentes continentes, já que as comparações realizadas pela equipe de Novotny foram entre Ásia tropical e Europa Central, enquanto que as realizadas pela equipe de Dyer foram entre diferentes localidades das Américas.

Esses estudos envolvem uma enorme complexidade de fatores, tornando generalizações mais difíceis de serem atingidas. Uma completa descrição da diversidade beta de uma determinada região precisa conter informações sobre as espécies de insetos de maior especificidade e sobre plantas hospedeiras mais raras, que são organismos mais difíceis de serem amostrados, levando a maioria dos estudos a se limitar a gêneros de plantas mais comuns, o que tende a diminuir valores de beta diversidade (Novotny et al. 2007). Um outro aspecto importante na comparação de dados entre as regiões tropical e temperada é a filogenia das espécies de plantas utilizadas nos estudos, uma vez que as comparações entre os trópicos e a região temperada devem considerar as diferenças na diversidade filogenética da vegetação (Novotny et al. 2006). Uma outra grande dificuldade encontrada nas generalizações sobre especificidade de hospedeiro de insetos fitófagos acontece quando comparamos diferentes guildas alimentares. Insetos fitófagos que se alimentam internamente, como galhadores, minadores e brocadores, tendem a ser mais especialistas do que fitófagos externos (Fowler 1996) e essas diferenças exigem cuidados nas comparações e generalizações sobre especificidade de hospedeiro de insetos fitófagos.

Além desses fatores, um outro grande problema encontrado em trabalhos que ponderam, além da especificidade, a abundância dos insetos fitófagos tropicais é a grande quantidade de espécies raras encontrada nas amostras (Price et al. 1995, Diniz \& Morais 1997, Novotny \& Basset 2000, Flinte et al. 2006). Novotny \& Basset (2000) ressaltam que a alta porcentagem de espécies raras pode ser, apenas, um artefato do método de amostragem, no caso de coletas que não excluem espécies "turistas" (que estão apenas repousando na planta, sem se alimentar) ou métodos que não amostram adequadamente o grupo de insetos fitófagos estudado. Essas espécies raras podem corresponder a: 1) espécies oligófagas que se alimentam da planta estudada, mas preferem outra espécie de planta próxima filogeneticamente, onde é mais abundante, 2) espécies generalistas que se encontram ocasionalmente na planta estudada, mas quando consideradas todas as plantas hospedeiras utilizadas pela espécie a sua abundância pode ser relativamente alta, ou ainda 3 ) espécies monófagas realmente raras. Novotny \& Basset (2000) observaram que quanto maior o número de espécies de plantas estudadas, menor a proporção de espécies com somente um indivíduo coletado ao longo do estudo (singletons). Esses autores concluem que a grande maioria das espécies raras encontradas corresponde a espécies oligófagas, que se alimentam da planta estudada, mas preferem outra espécie de planta onde são mais abundantes, e espécies generalistas, que se alimentam de um grande número de espécies de plantas, totalizando nessas plantas uma população relativamente abundante. Esses resultados mostram mais uma vez que o número ou a representatividade de espécies de plantas amostradas num estudo é importante em trabalhos sobre especificidade de insetos fitófagos tropicais.

\section{PADRÕES DE ESPECIFICIDADE DE INSETOS EM BIOMAS BRASILEIROS}

Os registros de plantas hospedeiras de insetos fitófagos acoplados a informações de biologia e comportamento desses insetos têm origem, no Brasil, nas décadas de 40 e 50, através dos trabalhos de Costa 
Lima, que resultaram na coleção "Insetos do Brasil" (1939 a 1962). Dividida em vários volumes, a obra traz informações sobre espécies de insetos de diversas ordens e suas plantas hospedeiras e é considerada, até hoje, a publicação com maior contribuição de registros de insetos fitófagos em suas plantas hospedeiras no Brasil. Mais tarde, em 1968, D'Araújo e Silva et al. publicaram um catálogo com inúmeras espécies de insetos fitófagos e suas respectivas plantas hospedeiras. Entretanto, esses trabalhos têm como fonte principal estudos pontuais ligados a alguns táxons de insetos, em geral, relacionados a pragas agrícolas ou vetores de doenças e, por isso, podem não refletir um padrão representativo da natureza.

Nas duas últimas décadas, começaram a surgir uma série de trabalhos nas regiões Sudeste e CentroOeste do Brasil, que avaliavam as plantas hospedeiras dos insetos fitófagos dessas regiões (Lewinsohn 1991, Diniz \& Morais 1995, Diniz \& Morais 1997, Andrade et al. 1999, Diniz \& Morais 2002). Em sua maioria, esses trabalhos representam inventários de insetos fitófagos em suas plantas hospedeiras, alguns deles com informações sobre abundância e especificidade desses insetos. Estudos realizados no cerrado de Brasília apresentam uma baixa proporção de plantas atacadas (Diniz \& Morais 1997) e uma alta especificidade dos insetos fitófagos (Diniz \& Morais 2002, Dyer et al. 2007). Entretanto, os locais de amostragem desses estudos eram as regiões de cerrado, um bioma com características peculiares em relação a sua vegetação e clima, tornando os resultados encontrados representativos somente para esse bioma.

Assim como nos estudos realizados em Papua Nova Guiné (Novotny \& Basset 2000), alguns estudos realizados no cerrado de Brasília apresentaram uma grande quantidade de espécies raras (Price et al. 1995, Diniz \& Morais 1997). Comparando os resultados obtidos no cerrado com os da região temperada, foram encontradas curvas de acumulação de espécies não assintóticas, maior riqueza de espécies, menor abundância das espécies e mais plantas ocupadas por insetos fitófagos no cerrado do que na região temperada. Esses estudos propõem esse padrão de alta riqueza de espécies raras para as comunidades de insetos fitófagos neotropicais, porém mais estudos em outros biomas dessa região precisam ser realizados para verificar se esse padrão se ajusta também para outros ecossistemas brasileiros.
Mais recentemente, alguns trabalhos vêm sendo realizados em formação vegetal de Restinga, dentro do bioma Mata Atlântica, analisando-se as plantas hospedeiras utilizadas pelos insetos fitófagos e verificando-se padrões de abundância desses insetos. Esses estudos apresentaram algumas diferenças em relação aos realizados no cerrado, como, por exemplo, uma alta porcentagem de plantas atacadas por insetos fitófagos (Flinte et al. 2006, Monteiro et al. 2007, Silveira et al. 2008), o que pode ser explicado pelas diferentes características vegetacionais e climáticas entre os dois sistemas. Algumas semelhanças também foram observadas, como a grande quantidade de espécies raras nas amostras. Porém, ainda é cedo para concluir sobre a semelhança ou não dos padrões ecológicos dos insetos nesses biomas. Em princípio, esses trabalhos mostram que a região neotropical pode ser mais heterogênea do que se imagina, tornando assim ainda mais difícil a generalização acerca de um padrão dominante para a região como um todo.

Lewinsohn \& Prado (2004), em importante trabalho sobre biodiversidade brasileira, descrevem o perfil do conhecimento sobre essa biodiversidade e ressaltam a grande quantidade de espécies ainda desconhecidas e o enorme esforço necessário para o preenchimento dessa lacuna. Os principais obstáculos descritos pelos autores para estimativas da diversidade brasileira são a existência de ecossistemas pouco explorados, o número limitante de especialistas, a dificuldade de identificação e a necessidade de revisão de grande parte dos grupos taxonômicos. Diante desse perfil, quaisquer conclusões a respeito da ecologia de insetos fitófagos neotropicais são ainda preliminares para essa região.

\section{CONSIDERAÇÕES FINAIS}

A especificidade de hospedeiro de insetos herbívoros é um tema importante dentro da ecologia de comunidades, uma vez que é um conhecimento essencial para as revisões das estimativas globais de número de espécies de insetos (Novotny et al. 2002). Neste tópico foram apresentadas e discutidas várias hipóteses que procuram explicar os mecanismos de manutenção da grande diversidade de insetos fitófagos (Basset 1992) e de plantas (Wright 2002) nos trópicos. Entretanto, cabe ressaltar que ainda são escassas as publicações sobre o assunto, particularmente em 
regiões tropicais, que sofrem ainda variações na escala de amostragem, nas características físicas dos biomas analisados e ainda nos grupos taxonômicos considerados.

Os trabalhos existentes indicam conclusões diferentes em relação à especificidade alimentar dos insetos fitófagos na região tropical. Entretanto, o conhecimento sobre ecologia de insetos tropicais ainda é extremamente incipiente e, desta forma, poucas informações são disponíveis a respeito da especificidade de hospedeiro dos insetos fitófagos tropicais (Janzen 1983, Basset 1992). Além disso, a maioria dos estudos que avaliam a abundância dos insetos herbívoros tropicais em cada espécie de planta hospedeira, normalmente, amostra somente uma parte pequena da flora local, coletando geralmente espécies de plantas pertencentes aos gêneros mais comuns localmente (Marquis 1991, Diniz \& Morais 1997, Novotny et al. 2002). Trabalhos que investigam grandes quantidades de plantas são, na maioria das vezes, apenas qualitativos, não considerando as densidades dos insetos fitófagos (Janzen 1980, 2003). Assim, o grande número de espécies de insetos fitófagos se alimentando de apenas uma espécie de planta hospedeira poderia ser reflexo da pequena porcentagem de plantas locais amostradas nesses estudos. O que podemos concluir é que os insetos fitófagos tropicais apresentam uma grande variação no grau de especificidade, tanto em localidades diferentes, quanto em guildas alimentares diferentes (Barone 1998, Marquis et al. 2002, Novotny $\&$ Basset 2005).

Uma vez que a riqueza de espécies é bem maior nos trópicos, mas o conhecimento sobre a ecologia dos insetos é ainda pequeno nessa região, faltam levantamentos e estudos sobre interações que permitam avaliar melhor as tendências das estimativas sobre diversidade global feitas anteriormente. Apesar de ainda serem necessários estudos que avaliem as densidades de insetos fitófagos tropicais e que amostrem uma grande quantidade de plantas hospedeiras locais, os estudos realizados até o momento têm revelado informações relevantes sobre a biologia reprodutiva das espécies e sobre outros aspectos relacionados à evolução de grupos de insetos e de associações co-evolutivas entre plantas e insetos fitófagos. Possivelmente, logo poderemos entender melhor a relação entre a diversidade de espécies encontrada em uma planta numa região e a biodiversidade de espécies dessa região.

\section{REFERÊNCIAS}

ANDRADE, I.; MORAIS, H.C.; DINIZ, I.R. \& VAN DEN BERG, C. 1999. Richness and abundance of caterpillars on Byrsonima (Malpighiaceae) species in an area of cerrado vegetation in Central Brazil. Revista de Biología Tropical, 47 (4): 691-695.

BARONE, J.A. 1998. Host-specificity of folivorous insects in a moist tropical forest. Journal of Animal Ecolology, 67: 400-409.

BARTHLOTT, W.; LAUER, W. \& PLACKE, A. 1996. Global distribution of species diversity in vascular plants: towards a world map of phitodiversity. Erdkunde, 50: 317-327.

BASSET, Y. 1992. Host specificity of arboreal and free-living insect herbivores in rain forests. Biological Journal of the Linnean Society, 47: 115-133.

BASSET, Y. 1996. Local communities of arboreal herbivores in Papua New Guinea: Predictors of insect variables. Ecology, 77 (6): 1906-1919.

BASSET, Y.; NOVOTNY, V.; MILLER, S.E. \& PYLE, R. 2000. Quantifying biodiversity: Experience with parataxonomists and digital photography in Papua New Guinea and Guyana. BioScience, 50 (10): 899-908.

COSTA LIMA, A. 1939-1962. Insetos do Brasil - 12 volumes. Escola Nacional de Agronomia, Rio de Janeiro.

D'ARAÚJO E SILVA, A.G.; GONÇALVES, C.R.; GALVÃO, D.M.; GONÇALVES, A.J.L.; GOMES, J.; SILVA, M.N. \& SIMONI, L. 1968. Quarto Catálogo dos Insetos que Vivem nas Plantas do Brasil, seus Parasitos e Predadores - Parte $I I-1^{\circ}$ tomo. Fundação IBGE, Rio de Janeiro. 622p.

DINIZ, I.R. \& MORAIS, H.C. 1995. Larvas de Lepidoptera e suas plantas hospedeiras em um cerrado de Brasília, DF, Brasil. Revista Brasileira de Entomologia, 39(4): 755-770.

DINIZ, I.R. \& MORAIS, H.C. 1997. Lepidopteran caterpillar fauna of Cerrado host plants. Biodiversity and Conservation, 6: $817-836$.

DINIZ, I.R. \& MORAIS, H.C. 2002. Local pattern of host plant utilization by lepidopteran larvae in the cerrado vegetation. Entomotropica, 17(2): 115-119.

DYER, L.A.; SINGER, M.S.; LILL, J.T.; STIREMAN, J.O.; GENTRY,G.L.; MARQUIS, R.J.; RICKLEFS, R.E.; GREENEY, H.F.; WAGNER, D.L.; MORAIS, H.C.; DINIZ, I.R.; KURSAR T.A. \& COLEY, P.D. 2007. Host specificity of Lepidoptera in tropical and temperate forests. Nature, 448: 696-700.

EHRLICH, P.R. \& RAVEN, P.H. 1964. Butterflies and plants: a study in coevolution. Evolution, 18: 586-608.

ERWIN, T.L. 1982. Tropical forests: their richness in Coleoptera and other species. Coleopterist's Bulletin, 36: 74-75. 
FLINTE, V.; ARAUJO, C.O.; MACEDO, M.V. \& MONTEIRO, R.F. 2006. Insetos fitófagos associados ao murici da praia, Byrsonima sericea (Malpighiaceae), na Restinga de Jurubatiba (RJ). Revista Brasileira de Entomologia, 50(4): 512-523.

FOWLER, H.G. 1996. Feeding guilds and host plant specificity in phytophagous insects. Journal of the Brazilian Association for the Advancement of Science, 48(3): 178-181.

GASTON, K.J. \& GAULD, I.D. 1993. How many species of pimplines (Hymenoptera: Ichneumonidae) are there in Costa Rica? Journal of Tropical Ecology, 9: 491-499.

HODKINSON, I.D. 1992. Global Insect Diversity Revisited. Journal of Tropical Ecology, 8 (4): 505-508.

HAMMOND, P.M. 1992. Species inventory. Pp17-39. In: B. Groombridge (ed.), Global biodiversity; status of the Earth's living resources. Chaoman and Hall, London. 594p.

HUTCHINSON, G.E. 1959. Homage to Santa Rosalia, or Why are there so many kinds of animals? American Naturalist, 93: 145-159.

JANZEN, D.H. 1980. Specificity of seed-attacking beetles in a Costa Rican deciduous forest. Journal of Ecology, 68: 929-952.

JANZEN, D.H. 1983. Food webs: who eats what, why, how, and with what effects in a tropical forest? Pp 167-182. In: F.B.I Golley (ed.), Tropical rain forest ecosystems. Elsevier, Amsterdam. 714p.

JANZEN, D.H. 1988. Ecological characterization of a Costa Rican dry forest caterpillar fauna. Biotropica, 20: 120-135.

JANZEN, D.H. 2003. How polyphagous are Costa Rican dry forest saturniid caterpillars? Pp 369-379. In: Y. Basset, V. Novotny, S.E. Miller \& R. Kitching (eds.), Arthropods of tropical forests: spatio-temporal dynamics and resource use in the canopy. Cambridge University Press, Cambridge. 474p.

LEWINSOHN, T.M. 1991. Insects in flower heads of Asteraceae in Southeast Brazil: a case study on tropical species richness. Pp 525-559. In: P.W. Price, T.M. Lewinsohn, G.W. Fernandes \& W.W. Benson (eds.), Plant-animal interactions: Evolutionary ecology in tropical and temperate regions. John Wiley, New York. 639p.

LEWINSOHN, T.M.; NOVOTNY, V. \& BASSET, Y. 2005. Insects on plants: Diversity of herbivores assemblages revisited. Annual Review of Ecology Evolution and Systematics, 36: 597-620.

LEWINSOHN, T.M \& PRADO, P.I. 2004. Biodiversidade brasileira - Síntese do estado atual do conhecimento. Contexto, São Paulo. 176p.

LEWINSOHN, T.M; PRADO, P.I.K.L. \& ALMEIDA, A.M. 2001. Inventários bióticos centrados em recursos: Insetos fitófagos e plantas hospedeiras. Pp 174-189. In: I. Garay \& B.
Dias (eds.), Conservação da Biodiversidade em Ecossistemas Tropicais. Editora Vozes, Petrópolis. 430p.

LOPES, B.C. 1995. Treehoppers (Homoptera: Membracidae) in southeastern Brazil: Use of host plants. Revista Brasileira de Zoologia, 12: 595-608.

MARQUIS, R.J. 1991. Herbivore fauna of Piper (Piperaceae) in a Costa Rican wet forest: Diversity, specificity, and impact. Pp 179-208. In: P.W. Price, T.M. Lewinsohn, G.W. Fernandes \& W.W. Benson (eds.), Plant-animal interactions: Evolutionary ecology in tropical and temperate regions. John Wiley, New York. 639p.

MARQUIS, R.J.; MORAIS, H.C. \& DINIZ, I.R. 2002. Interactions among cerrado plants and their herbivores: unique or typical? Pp 306-328. In: P.S. Oliveira \& R.J. Marquis (eds.), The cerrados of Brazil: ecology and natural history of a Neotropical savanna. Columbia University Press, New York. 424p.

MAY, R.M. 1988. How many species are there on Earth? Science, 241 (4872): 1441-1449.

MONTEIRO, R.F.; MACEDO, M.V.; NASCIMENTO, M.S. \& CURY, R.S.F. 2007. Composição, abundância e notas sobre a ecologia de espécies de larvas de lepidópteros associadas a cinco espécies de plantas hospedeiras no Parque Nacional da Restinga de Jurubatiba, RJ. Revista Brasileira de Entomologia 51(4): 313-320.

NOVOTNY, V. \& BASSET, Y. 2000. Rare species in communities of tropical insect herbivores: pondering the mystery of singletons. Oikos 89(3): 564-572.

NOVOTNY, V. \& BASSET, Y. 2005. Host specificity of insect herbivores in tropical forests. Proceedings of the Royal Society, 272: 1083-1090.

NOVOTNY, V.; BASSET, Y.; MILLER, S.E.; WEIBLEN, G.D; BREMER, B.; CIZEK, L. \& DROZD, P. 2002. Low host specificity of herbivorous insects in a tropical forest. Nature, 416: 841-844.

NOVOTNY, V.; CLARKE, A.R.; DREW, R.A.I.; BALAGAWI, S. \& CLIFFORD, B. 2005. Host specialization and species richness of fruit flies (Diptera: Tephritidae) in a New Guinea rain forest. Journal of Tropical Ecology, 21: 67-77.

NOVOTNY, V.; DROZD, P.; MILLER, S.E.; KULFAN, M.; JANDA, M.; BASSET, Y. \& WEIBLEN, G.D. 2006. Why are there so many species of herbivorous insects in Tropical Rainforests? Science, 313: 1115-1118.

NOVOTNY, V.; MILLER, S.E.; HULCR, J.; DREW, R.A.I.; BASSET, Y.; JANDA, M.; SETLIFF, G.P.; DARROW, K.; STEWART, A.J.A.; AUGA, J.; ISUA, B.; MOLEM, K.; MANUMBOR, M.; TAMTIAI, E.; MOGIA, M. \& WEIBLEN, G.D. 2007. Low beta diversity of herbivorous insects in tropical forests. Nature, 448: 692-695. 
NOVOTNY, V.; MILLER, S.E.; LEPS, J.; BITO, D.; JANDA, M.; HULCR, J.; BASSET, Y.; DAMAS, K. \& WEIBLEN, G.D. 2004. No tree an island: the plant-caterpillar food web of secondary rainforest in New Guinea. Ecology Letters, 7: $1090-1100$

ODEGAARD, F. 2000. How many species of arthropods? Erwin's estimate revised. Biological Journal of the Linnean Society, 71: 583-597.

ORR, A.G. \& HAEUSER, C.L. 1996. Temporal and spatial patterns of butterfly diversity in a lowland tropical rainforest. Pp 125-138. In: D.S. Edwards, W.E. Booth \& S. Choy (eds.), Tropical Rainforest Research — Current Issues. Kluwer, Dordrecht. 584p.

PRICE, P.W. 1984. Communities of specialists: vacant niches in ecological and evolutionary time. Pp 510-523. In: D.R. Strong, D. Simberloff, L. Abele \& A.B. Thistle (eds.), Ecological communities: conceptual issues and the evidence. Princeton University Press, New Jersey. 613p.

PRICE, P.W.; DINIZ, I.R.; MORAIS, H.C. \& MARQUES, E.S.A. 1995. The abundance of insect herbivore species in the tropics: high local richness of rare species. Biotropica, 27: 468-478.

QUINN, R.M.; GASTON, K.J.; ROY, D.B. 1997. Coincidence between consumer and host occurrence: macrolepidoptera in Britain. Ecological Entomology, 22: 197-208.

SILVEIRA, V.R.; MONTEIRO, R.F \& MACEDO, M.V. 2008. Espécies de larvas de insetos associadas à Clusia hilariana Schltdl. (Clusiaceae) na Restinga de Jurubatiba, RJ-Brasil. Revista Brasileira de Entomologia, 52: 57-61.

STIREMAN, J.O.; NASON, J.D. \& HEARD, S.B. 2005. Hostassociated genetic differentiation in phytophagous insects: General phenomenon or isolated exceptions? Evidence from a Goldenrod-insect community. Evolution, 59(12): 2573-2587.

STORK, N.E. 1988. Insect diversity: Facts, fiction and speculation. Biological Journal of the Linnean Society, 35 (4): 321-337.

STORK, N.E. 1993. How many species are there? Biodiversity Conservation, 2: 215-232.

STRONG, D.R. 1984. Exorcising the gost of competition past: phytophagous insects. Pp 28-41. In: D.R. Strong, D. Simberloff, L. Abele \& A.B. Thistle (eds.), Ecological communities: conceptual issues and the evidence. Princeton University Press, New Jersey. 613p.

THORSTEINSON, A.J. 1960. Host selection in phytophagous insects. Annual Review of Entomology, 5: 193-218.

WRIGHT, S.J. 2002. Plant diversity in tropical forests: a review of mechanisms of species coexistence. Oecologia, 130: 1-14. 